\title{
Patrones de cacería y consumo de proteína animal en cuatro comunidades Waorani, Reserva de Biósfera Yasuní
}

\author{
Hunting patterns and animal protein consumption in four communities \\ Waorani, Yasuní Biosphere Reserve
}

Sulaya Betsabé Bayancela Delgado ${ }^{1}$

Recibido: 18-05-2019 / Revisado: 17-06-2019 /Aceptado: 06-07-2019/ Publicado: 28-07-2019

\begin{abstract}
.
DOI: https://doi.org/10.33262/cienciadigital.v3i3.2.1.781

This study assesses the main hunting activities in four Waorani communities located at different levels of access from Puerto Francisco de Orellana (El Coca) within the Yasuní Biosphere Reserve (Napo, Pastaza, and Orellana, Ecuador). More specifically, the removal of animal biomass, type of species, use of weapons, and preferred animal are discussed. This study describes the frequency of consumption of animal protein acquired in and out of the observed communities, and their relation to the levels of access. For two months, I interviewed the heads (hunters) of 44 families and observed their hunting activities. Results show that hunting practices vary in relation to the level of access to El Coca. Furthermore, bushmeat is consumed more in the isolated community (Bameno) than in the others, possibly due to a lower percentage of wage labor. The frequency of consumption of wild fish in all communities is constant and is consumed 2 to 3 days per week. Canned fish (tuna, sardines) is consumed mostly in communities with a high percentage of wage labor, independently of level of access. Fresh purchased chicken and fish are consumed 1 to 2 days per week in communities that are close to El Coca.
\end{abstract}

Keywords: Hunting, Waorani, Yasuni, animal protein.

\footnotetext{
${ }^{1}$ Escuela Superior Politécnica de Chimborazo, Facultad de Recursos Naturales. Riobamba, Ecuador. sulaya.bayancela@espoch.edu.ec
} 


\section{Resumen.}

El presente estudio evalúa las principales actividades de cacería (extracción de biomasa animal, tipo de especie, uso de armas y animal apetecible) en cuatro comunidades Waorani ubicadas a diferentes niveles de acceso a Puerto Francisco de Orellana (El Coca), dentro de la Reserva de Biósfera Yasuní (Napo, Pastaza y Orellana, Ecuador), y describe las frecuencias de consumo de proteína animal adquirida dentro y fuera de las comunidades observadas, y su relación con los niveles de acceso. Durante dos meses, se realizaron entrevistas a los jefes de hogar (cazadores) de 44 familias y se observaron sus actividades de cacería. Los resultados muestran que las prácticas de cacería varían en relación con el nivel de acceso a El Coca. Asimismo, el consumo de carne de monte es mayor en la comunidad de difícil acceso (Bameno); posiblemente debido al bajo porcentaje de trabajo asalariado. La frecuencia de consumo de pescado silvestre en todas las comunidades es constante, éste es consumido entre 2 y 3 días por semana. El pescado enlatado (atún y sardina) es consumido mayormente en comunidades con alto porcentaje de trabajo asalariado, independientemente del nivel de acceso. La carne comprada (pollo y pescado fresco) es consumida entre 1 y 2 días por semana en comunidades que están cerca de El Coca.

Palabras claves: Cacería, Waorani, Yasuní, proteína animal.

\section{Introducción.}

La caza es una actividad de subsistencia entre los grupos indígenas amazónicos. Hasta el 90\% de la proteína consumida por algunas familias en la selva amazónica (Redford, 1991) proviene de la carne de animales silvestres. La caza también es una de las principales causas de la disminución y el agotamiento de la vida silvestre local (Cueva, Ortiz y Jorgenson, 2004; Mena, Stallings, Regalado y Cueva, 2000; Vickers, 1989; Quirós-Fernández et al., 2017), dando lugar al llamado "síndrome del bosque vacío", el cual consiste en la eliminación de la mayoría de los vertebrados salvajes (Redford y Robinson, 1987). Los pueblos indígenas amazónicos tienen una visión amplia del contexto ecológico donde viven, permitiéndoles hacer uso de los recursos naturales para sobrevivir (Cueva et al., 2004).

Los habitantes de la Amazonía cambiaron su forma de vida para adaptarse a las nuevas condiciones que surgieron con la llegada de nuevos elementos de la cultura occidental (Borgerson, 2015). La selva amazónica es un recurso natural de gran valor porque alberga una gran cantidad de especies florísticas y faunísticas. Además, la Amazonía brinda servicios ambientales como el suministro de agua dulce, purifica el aire, regula el clima, proporciona oxígeno, mantiene los recursos genéticos, preserva los valores intrínsecos ecológicos, socioculturales, estéticos y económicos (Ortiz-von Halle, 2011), suministra alimentos, medicina ancestral y vivienda para los pueblos indígenas de esta región, y protege la vida silvestre (Redford, 1991). 
Diferentes grupos étnicos viven en la selva, incluidos los Waorani que habitan la Reserva de Biósfera Yasuní (Bryja, 2009), ellos, desde su contacto inicial con misioneros evangélicos en 1958 incorporaron en su cultura, elementos occidentales como ropa, vivienda, creencias religiosas, alimentos externos, entre otros (Rival 2001). Los Waorani tienen el territorio indígena más grande del país $(613,750$ hectáreas) que incluye parte del Parque Nacional Yasuní y la Zona Intangible Tagaeri Taromenane (Lara et al., 2002).

En su territorio, los Waorani son principalmente cazadores, su alimentación se basa de proteína animal silvestre; especialmente de mamíferos grandes (Robinson y Redford, 1987, Mena et al. 1997, Mena et al. 2000, Vickers 1989). Usan armas tradicionales para cazar: lanzas y cerbatanas. Sin embargo, después de la llegada de misioneros evangélicos (Cabodevilla, 1995; Rival, 2001), el uso de armas de fuego introducidas como escopetas y machetes ha aumentado considerablemente (Mena-Valenzuela, Regalado y Cueva, 1997; Mena et al., 2000).

Varios estudios (Franzen y Eaves, 2007; Lu, 2010; Mena-Valenzuela et al., 1997; Mena y Cueva, 2001; Urgilés, 2006; Vickers, 1989; Zapata-Ríos, Suárez, Utreras y Vargas, 2006; Zapata Ríos, Toasa, Neill y Jørgenson, 2004; Da Silva Neto et al., 2017) han evaluado la subsistencia de cacería (oferta y la demanda de animales).

Sin embargo, hay poca investigación sobre las frecuencias del consumo de proteína en la dieta Waorani. Por lo tanto, evaluar las prácticas de cacería permitirá conocer las presiones actuales sobre las especies afectadas para definir estrategias de conservación. Asimismo, obtener datos sobre las frecuencias de consumo de proteína animal ayudará a reconocer la demanda actual requerida por las familias Waorani.

Esta información permitirá reducir la caza de animales cuando se satisface la necesidad de proteínas en la dieta alimenticia. Los objetivos del presente estudio fueron: i) evaluar los patrones de caza en cuatro comunidades Waorani ubicadas a diferentes niveles de acceso a la ciudad de El Coca, categorizando las comunidades según la distancia y el tiempo requerido para viajar por tierra y río desde la comunidad hasta el mercado en El Coca.

Los patrones de cacería considerados fueron: cantidad de biomasa silvestre (kilogramos), especie, arma utilizada y animal apetecible para consumo; ii) describir las frecuencias de consumo de la proteína animal adquirida dentro y fuera de cada comunidad.

Los patrones de consumo considerados fueron: frecuencia de consumo de proteína animal según su origen: interna (pescado silvestre y carne de monte), y externa (carne comprada y enlatados). Finalmente, el porcentaje de trabajo remunerado de los cazadores en relación a los niveles de accesibilidad a la ciudad de El Coca. 


\section{Métodos}

\section{Área de estudio}

Los Waorani viven en el lugar más biodiverso del planeta (Bass et. al 2010) y tienen la mayor extensión de territorio indígena del país "La Reserva Étnica Waorani" en la cual existen 48 asentamientos, el acceso a éstos es muy limitado, para llegar se requiere viajar días en canoa, o ir en avioneta; los Waorani son llamados peyorativamente "aucas" que significa "salvajes"; en 1956, tuvieron su primer contacto con cinco misioneros evangélicos del Instituto Lingüístico de Verano, la avioneta del ILV aterrizó en una playa del río Curaray, los cinco misioneros murieron en su intento de hacer contacto (Lara et al. 2002). En 1958, Raquel Saint y Elizabeth Elliot, misioneras del ILV, crearon la primera misión evangélica Waorani a orillas del río Tiweno con la ayuda de mujeres Waorani; así se establece el enlace entre los mundos Waorani y occidental (Cabodevilla1994). La reserva indígena se denominó el "Protectorado de Tiweno" (Trujillo 2011). Para los actores políticos y sociales, los Waorani son indígenas que se caracterizan por ser guerreros, agresivos e incivilizados, y entrar en contacto con ellos se torna muy difícil.

Las comunidades Waorani seleccionadas fueron: Bameno, Keweriono, Ñoneno y Tiwino, ubicadas dentro de la Reserva de Biosfera Yasuní (RBY) (Figura 1). La historia de la Reserva comenzó en 1979 cuando el gobierno nacional estableció el Parque Nacional Yasuní con un área de 678,000 hectáreas. En 1989, la UNESCO lo declaró Reserva de la Biósfera con 2.740.409 hectáreas. Y, en 1999, se estableció la Zona Intangible Tagaeri-Taroemane con 752,718 hectáreas (Lara et al., 2002). La Reserva, ubicada en las provincias de Pastaza, Napo y Orellana, tiene un clima cálido y húmedo, una altitud entre 200 y 300 metros sobre el nivel del mar y una precipitación de $3.000 \mathrm{~mm}$ por año.

Mantiene una temperatura de 24 a $26^{\circ}$ C. Napo, Shiripuno, Curaray, Tiputini, Yasuní y Cononaco son los principales ríos de la reserva. La zonificación del RBY incluye: i) la zona central intangible (Tagaeri-Taromenane, Yasuní y Waorani) creada para proteger a los indígenas que viven en aislamiento voluntario, ii) la zona de amortiguamiento y iii) la zona de transición que incluye el camino Auca que comienza en la ciudad de El Coca y se extiende por $105 \mathrm{~km}$. hasta la comunidad de Tiwino. La carretera Auca se construyó entre 1970 y 1980 (Jorgenson y Rodríguez, 2001) para facilitar la explotación petrolera en la región central del Amazonas (Villaverde, 2005).

Ochenta y dos kilómetros del camino han sido pavimentados, y el resto es grava. A lo largo de la carretera, hay largos oleoductos y chimeneas en llamas por la quema de gas. Para clasificar el nivel de accesibilidad de las comunidades, se consideró distancia y tiempo de viaje por tierra y río desde cada comunidad hasta el mercado en El Coca. Así, las categorías fueron: fácil acceso, Tiwino (283298,31; 9875136,59 UTM) y Ñoneno (290149,34; 
9884544,88), medio acceso, Keweriono (258184,46; 9887194), y difícil: Bameno $(371346,87 ; 9856235,48)$.

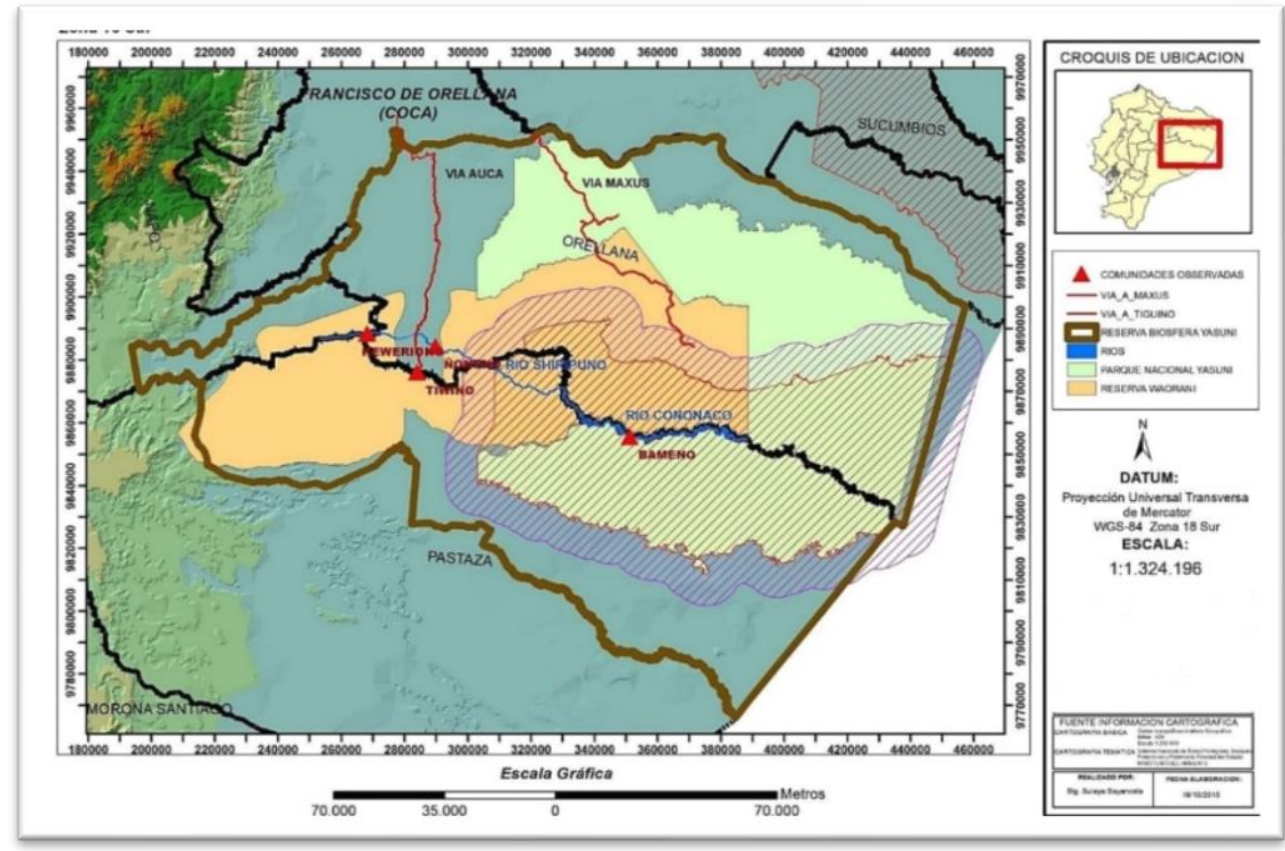

Figura 1. Área de estudio

Elaborado por: Investigadora

\section{Recolección de datos}

Para evaluar los patrones de cacería y consumo de alimentos en las comunidades Waorani se realizaron encuestas a 44 jefes de hogar (cazadores) y se observaron las actividades de cacería de las familias, de mayo a julio de 2013.

\section{Encuestas}

Los cuarenta y cuatro jefes de hogar (cazadores) corresponden al 50\% de las familias de las cuatro comunidades (Tiwino $n=20$, Keweriono, $n=10$; Noneno, $n=7$; $y$ Bameno, $n=7$ ). Fueron seleccionados según la apertura y disposición del cazador. Un miembro de cada comunidad colaboró como traductor. Antes de la fase de campo, se obtuvo el permiso del Comité de Bioética de la Universidad San Francisco de Quito. En consecuencia, se buscó el consentimiento informado de los entrevistados antes de su participación.

Se elaboró la encuesta estructurada, se registró:

- Demografía: nombre de la comunidad, tamaño de la familia, código del cazador (a cada cazador se le asignó un código para ocultar sus nombres y obtener el permiso del Comité de Bioética), edad y trabajo asalariado actual.

- Accesibilidad de la comunidad a El Coca: costo en dólares por cada viaje, frecuencias de salidas realizadas durante el último mes y horas de viaje. 
- Frecuencia de consumo de proteína animal interna y externa. Se aplicó el cuestionario de frecuencia de consumo de alimentos (FFQ); Las categorías se establecieron en días por semana: 6 a 7 días, 3-5 días, 1 a 2 días y 0 días (Stefanik y Trulson, 1962).

\section{Observación directa}

Para evaluar las prácticas de cacería, se elaboró una ficha de observación con los siguientes datos: nombre común del animal, especie, edad relativa, arma usada y peso estimado en kilogramos. Cuando fue posible el animal capturado fue pesado, para aquel que no pudo ser pesado, se usó los pesos promediados reportados en el libro Mamíferos del Ecuador (Tirira 2017). Para identificar a los animales se utilizaron las guías de campo del Ecuador: mamíferos (Tirira 2017), aves (Navarrete y McMullan 2018) y reptiles (Valencia 2008), guías que sirvieron también para identificar el animal apetecible para consumo por los cazadores; además, se participó en dos eventos de cacería por cada comunidad, desde las 0600 hasta las 1600. Se caminó entre 6 a 8 horas.

\section{Resultados}

\section{Prácticas de cacería: Biomasa y composición de la caza.}

Los Waorani cazaron $964 \mathrm{~kg}$. Bameno, comunidad de difícil acceso registró $372.5 \mathrm{~kg}$; Keweriono, acceso medio, $247 \mathrm{~kg}$; las comunidades de fácil acceso, Tiwino $232 \mathrm{~kg}$, y Noneno $112.5 \mathrm{~kg}$. Las especies con mayor biomasa fueron: Tayassu pecari (pecarí de labio blanco $332 \mathrm{~kg}$ ), Tapirus terrestres (tapir $230 \mathrm{~kg}$ ), Pecari tajacu (pecarí con collar $219 \mathrm{~kg}$ ), Lagothrix lagothricha poeppigii (mono lanudo 47,2 kg), Dasyprocta fuliginosa (guatusa 22,3 $\mathrm{kg}$ ), Ateles belzebuth (mono araña $21.2 \mathrm{~kg}$ ) y Pipile cumanensis (pava azul $17.1 \mathrm{~kg}$ ). Los ungulados representaron el 81\% (781 kg) de la biomasa; en Bameno atraparon 38 individuos; Noneno 11; Tiwino 22 y Keweriono 5. En total, cazaron 40 mamíferos, 33 aves y 3 reptiles que pertenecen a 18 especies (Tabla 1 ).

Tabla 1. Animales cazados por comunidad: cantidad y peso

\begin{tabular}{|c|c|c|c|c|c|c|c|c|c|c|}
\hline Especies & (No.) & $\begin{array}{l}\text { Bameno } \\
\text { (kg) }\end{array}$ & $\begin{array}{c}\text { (No. } \\
\text { ) }\end{array}$ & $\begin{array}{l}\text { Ñ̃oneno } \\
\text { (kg) }\end{array}$ & $\begin{array}{l}\text { (No } \\
\text {.) }\end{array}$ & $\begin{array}{l}\text { Tiwino } \\
\text { (kg) }\end{array}$ & $\begin{array}{c}\text { (No. } \\
\text { ) }\end{array}$ & $\begin{array}{l}\text { Keweriono } \\
\text { (kg) }\end{array}$ & \begin{tabular}{|l|} 
Total \\
$(\mathrm{kg})$
\end{tabular} & $\begin{array}{l}\text { Total } \\
\text { (No.) }\end{array}$ \\
\hline Accesibilidad & & Difícil & & Fácil & & Fácil & & Media & & \\
\hline \multicolumn{11}{|l|}{ Primates } \\
\hline $\begin{array}{l}\text { Lagothrix lagothricha } \\
\text { poeppigii }\end{array}$ & 3 & 24,3 & 3 & 22,9 & & & & & 47,2 & 6 \\
\hline Ateles belzebuth & 4 & 21,2 & & & & & & & 21,2 & 4 \\
\hline Alouatta seniculus & 1 & 7,5 & & & 1 & 7,3 & & & 14,8 & 2 \\
\hline \multicolumn{11}{|l|}{ Ungulate } \\
\hline Tayassu pecari & 7 & 196 & 2 & 67 & 2 & 69 & & & 332 & 11 \\
\hline Pecari tajacu & 4 & 98 & & & 5 & 121 & & & 219 & 9 \\
\hline Tapirus terrestris & & & & & & & 1 & 230 & 230 & 1 \\
\hline Carnívores & & & & & & & & & & \\
\hline
\end{tabular}


ISSN: 2602-8085

WWW.cienciadigital.org

Vol. 3, N³.2.1, p. 43-60, julio, 2019

\begin{tabular}{|c|c|c|c|c|c|c|c|c|c|c|}
\hline Nasu nasua & & & & & 1 & 5,1 & & & 5,1 & 1 \\
\hline \multicolumn{11}{|l|}{ Rodentios } \\
\hline Dasyprocta fuliginosa & & & 4 & 17,8 & 1 & 4,5 & & & 22,3 & 5 \\
\hline Cuniculus paca & & & & & & & 1 & 8,2 & 8,2 & 1 \\
\hline \multicolumn{11}{|l|}{ Reptiles } \\
\hline \begin{tabular}{|l|}
$\begin{array}{l}\text { Chelonoides } \\
\text { denticulada }\end{array}$ \\
\end{tabular} & & & & & 1 & 7,3 & & & 7,3 & 1 \\
\hline \begin{tabular}{|l|} 
Paleosuchus \\
trigonatus
\end{tabular} & & & & & 1 & 6,8 & 1 & 6,8 & 13,6 & 2 \\
\hline \multicolumn{11}{|l|}{ Birds } \\
\hline Pipile cumanensis & 15 & 17,1 & & & & & & & 17,1 & 15 \\
\hline Ara ararauna & 3 & 5,4 & & & & & & & 5,4 & 3 \\
\hline Penelope jacquacu & & & 1 & 3,6 & 1 & 3,6 & & & 7,2 & 2 \\
\hline Ortalis guttata & & & & & 1 & 1 & 2 & 2 & 3 & 3 \\
\hline Mitu salvini & 1 & 3 & & & & & & & 3 & 1 \\
\hline Selenidera reinwardtii & & & & & 8 & 6,4 & & & 6,4 & 8 \\
\hline Psophia crepitans & & & 1 & 1,2 & & & & & 1,2 & 1 \\
\hline Number of species & 8 & & 5 & & 10 & & 4 & & & \\
\hline Total & 38 & 372,5 & 11 & 112,5 & 22 & 232 & 5 & 247 & 964 & 76 \\
\hline
\end{tabular}

Elaborado por: Investigadora

Estado de conservación de algunas especies cazadas durante el estudio.

Según la Lista Roja de Especies en Peligro de Extinción de la Unión Mundial para la Naturaleza (Unión Internacional para la Conservación de la Naturaleza -UICN, 2018), de los animales capturados en las cuatro comunidades, cinco especies están en alguna categoría de amenaza en todo el mundo. Estas especies son: mono araña en peligro de extinción (EN), de vientre amarillo; Vulnerable (VU), mono lanudo, tapir, pecarí de labios blancos (UICN, 2018) y la tortuga de patas amarillas (UICN 1996); casi amenazado (LC), trompetista de alas grises (UICN, 2018). Según la Lista Roja de Ecuador, el mono araña de vientre blanco y el mono lanudo se consideran especies en peligro de extinción (EN) (Papworth, Milner-Gulland y Slocombe, 2013; Tirira, 2017).

\section{Arma usada}

En Bameno, comunidad de difícil acceso, se utilizó la cerbatana 26.3\%; En las tres comunidades, el uso de armas tradicionales disminuyó (Figura 2). Las armas de fuego fueron las más utilizadas en todas las comunidades, excepto Tiwino, donde se usó el machete. Mientras que los cazadores en Keweriono solo usaron armas de fuego y machetes. 
Figura 1. Porcentaje de animales cazados por tipo de arma

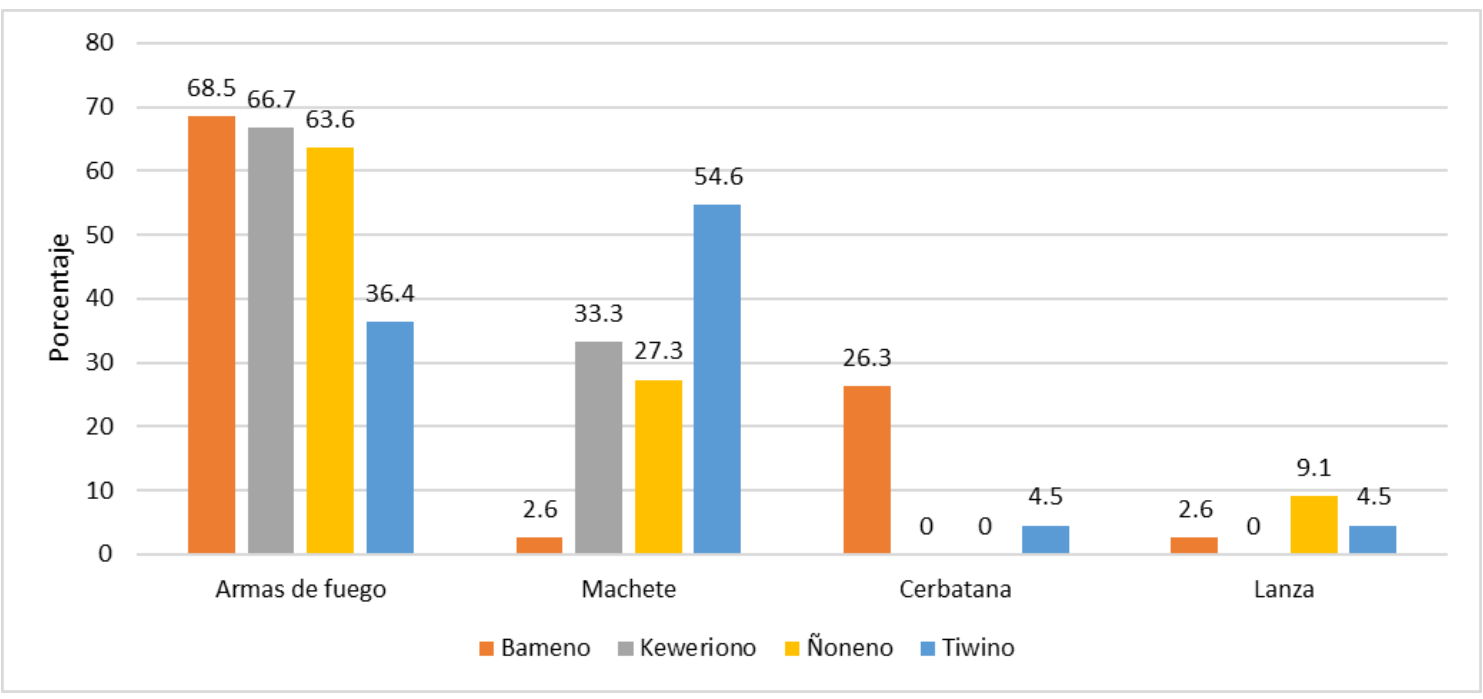

Elaborado por: Investigadora

Además, el $88.3 \%$ de todos los animales capturados en las cuatro comunidades fueron cazados con armas introducidas (58.8\% de armas de fuego y $29.5 \%$ de machete) y el $11.7 \%$ con armas tradicionales ( $7.7 \%$ de cerbatana y $4 \%$ de lanza) (Tabla 2$)$.

Tabla 2. Cantidad en porcentaje de animales cazados según el arma (\%)

\begin{tabular}{llllll}
\hline Comunidad & $\begin{array}{l}\text { Animal } \\
\text { Cazado }\end{array}$ & $\begin{array}{l}\text { Arma de } \\
\text { fuego }\end{array}$ & Machete & Cerbatana & Lanza \\
\hline Bameno & 38 & 68,5 & 2,6 & 26,3 & 2,6 \\
Keweriono & 6 & 66,7 & 33,3 & 0 & 0 \\
Ñoneno & 11 & 63,6 & 27,3 & 0 & 9,1 \\
Tiwino & 21 & 36,4 & 54,6 & 4,5 & 4,5 \\
\hline Promedio & $\mathbf{1 9}$ & $\mathbf{5 8 , 8}$ & $\mathbf{2 9 , 5}$ & $\mathbf{7 , 7}$ & $\mathbf{4}$ \\
\hline
\end{tabular}

Elaborado por: Investigadora

La biomasa obtenida según el arma utilizada fue: armas de fuego $807.86 \mathrm{~kg}$, machete 70.4 $\mathrm{kg}$, lanza $54.5 \mathrm{~kg}$, cerbatana $31.24 \mathrm{~kg}$. (Figura 3). 
Figure 2. Biomasa (Kilogramos) de los animales cazados

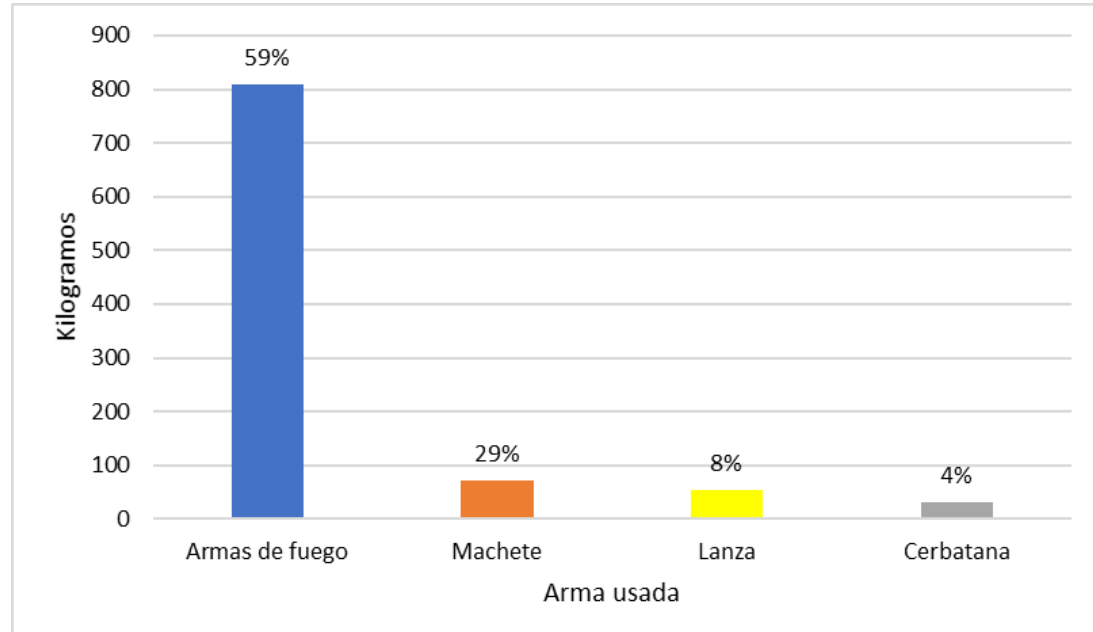

Elaborado por: Investigadora

\section{Animal apetecible}

La evaluación del animal apetecible se basa en (Rival, 1992) sobre la importancia social de las elecciones tecnológicas de los Waorani. El animal es relativamente apreciado por su sabor, carne, suavidad, grasa y por la facilidad de obtenerlo (Stafford, Preziosi y Sellers, 2017).

En Bameno les apetece el Tayassu pecari (pecarí de labio blanco-71\%) y Pecari tajacu (pecarí de collar-29\%); en Tiwino: Pecari tajacu (70\%); en Ñoneno: pecarí de labio blanco (57\%), y en Keweriono: Lagothrix lagothricha poeppigii (mono lanudo-50\%). (Figura 4). Después de los pecaríes, los monos son una de las presas más buscadas a pesar de tener una carne dura, musculosa y fibrosa (Rival, 1992).

Figura 3. Animal apetecible por porcentaje

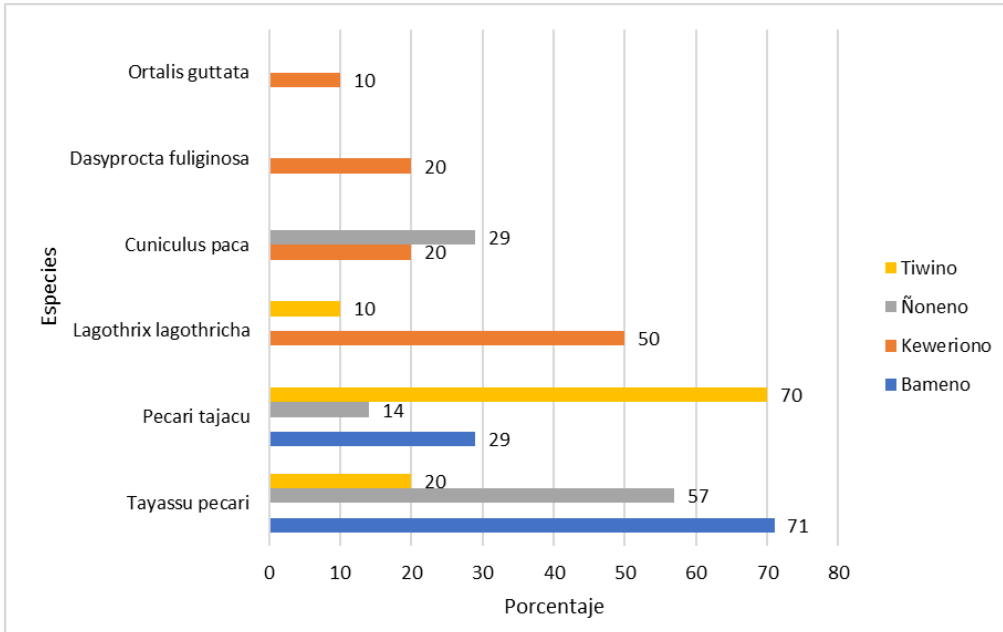

Elaborado por: Investigadora 


\section{Frecuencias de consumo de proteína animal}

En Bameno, las familias consumen carne de monte 3 días/semana, al igual que el pescado, no consumen enlatados. En Keweriono, la carne de monte es consumida 1 día/semana y el pescado silvestre 3 días/semana; enlatados 1 día/semana y la carne comprada no se consume. En Ñoneno, la carne de monte se consume 2 día/semana, al igual que el pescado, la carne comprada 1 día/semana, mientras que enlatados de pescado no se consume. En Tiwino, la carne de monte se consume 1 día/semana, el pescado silvestre 2 días/semana, enlatados de pescado 1 día/semana y la carne comprada 2 días/semana. Tiwino es la única comunidad que consume enlatados y carne comprada 3 días a la semana (Figura 5).

Figura 5. Frecuencia (días/semana) de proteína animal consumida por familias y comunidad

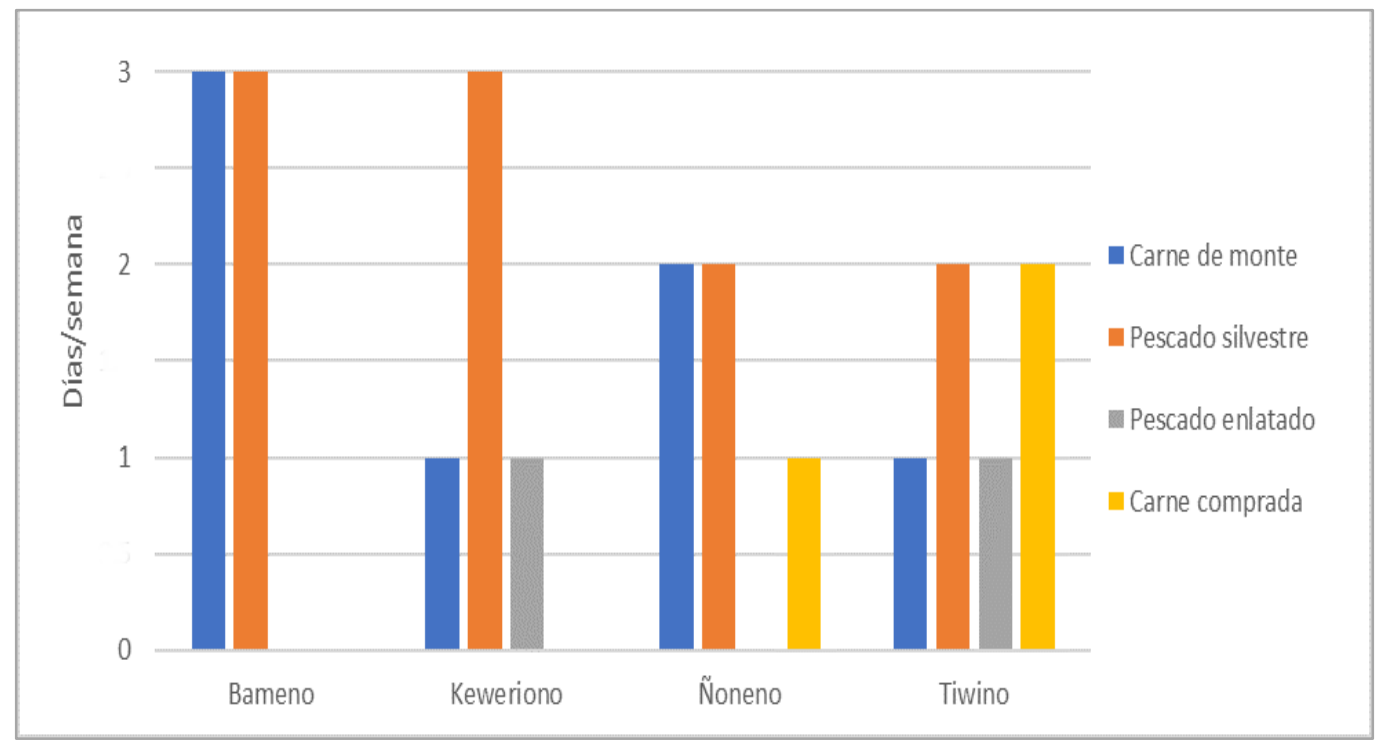

Elaborado por: Investigadora

\section{Familias y trabajo remunerado}

Una familia típica tiene un promedio de seis miembros. La edad promedio de los cazadores era de 39 años $(n=44$, rango 19-60) y el $85.5 \%$ tenía trabajo remunerado (Figura 6). En todas las comunidades, el porcentaje de trabajo remunerado por sector productivo fue: turismo $44.9 \%$, construcción $13.75 \%$, sector público (docentes, monitores comunitarios, políticos) $12.15 \%$, artesanía $7.33 \%$ y empresas petroleras $7.33 \%$. Y, el $71.4 \%$ de los cazadores de la comunidad de difícil acceso tenía trabajo remunerado. 
Figura 6. Porcentaje de cazadores con trabajo remunerado en las cuatro comunidades Waorani

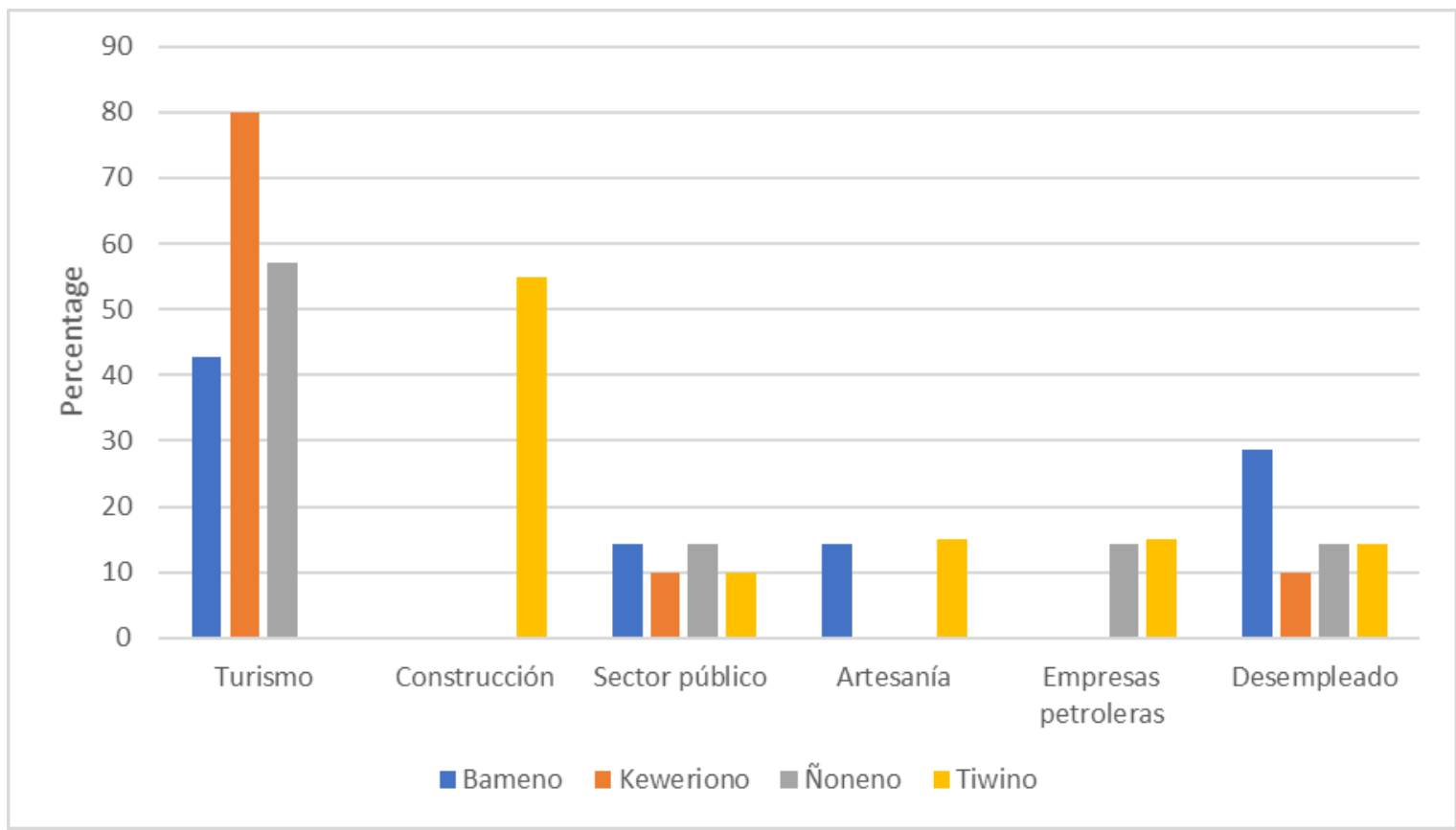

Elaborado por: Investigadora

\section{Discusión}

Los datos sugieren que las prácticas de cacería (extracción de especies, biomasa animal, uso de armas y animal apetecible) y las frecuencias de consumo de carne de monte y carne comprada están relacionadas con la accesibilidad de la comunidad. El porcentaje de trabajo remunerado es mayor en comunidades de fácil acceso en comparación con aquellas de difícil acceso. La cantidad de biomasa animal silvestre fue mayor en la comunidad de difícil acceso porque el bosque está en buenas condiciones. Además, el bosque circundante de la comunidad de difícil acceso se encuentra dentro de la Zona Intangible del Parque Nacional Yasuní, lo que aumenta la posibilidad de encontrar animales grandes. Así, en Bameno, cazaron $372.5 \mathrm{~kg}$. En las comunidades de fácil acceso, cazaron: Tiwino $112.5 \mathrm{~kg}$ y Ñoneno $232 \mathrm{~kg}$-en comunidades cercanas a las carreteras, los animales grandes son escasos (Franzen y Eaves, 2007; Suárez, Zapata-Ríos, Utreras, Strindberg y Vargas, 2013), y cazar animales pequeños como guatusas negras, coatí sudamericano y paca de tierras bajas es más fácil porque estos animales visitan granjas para alimentarse (Mena et al. 2001, Cueva et al., 2003). El nivel de acceso de una comunidad al mercado de alimentos varía en relación a la biomasa animal silvestre consumida. Esto coincide con investigaciones previas sobre cacería (Cueva 
et al., 2004; Franzen \& Eaves, 2007) y la demanda de animales (Mena-Valenzuela et al., 1997). Estos autores describen que animales de gran tamaño y peso (biomasa) como pecaríes y tapires son los más susceptibles de ser cazados en comunidades cercanas a los mercados; Sin embargo, deben considerarse otros factores como la edad del asentamiento (Franzen, 2006), la historia de la explotación de los recursos de fauna y la demografía de la comunidad (Da-Gloria y Bueno, 2018). Las especies cazadas registradas son diferentes por cada comunidad; en Bameno cazaron tres especies de primates, en Noneno una, en Tiwino y Keweriono ninguna. Además, se capturaron diferentes especies de aves en las comunidades estudiadas. En Bameno, se cazó pava de garganta azul, paujil y guacamayos; mientras que estas especies no se registraron en las otras comunidades; podría deberse a que, con el tiempo, las especies vulnerables y grandes que habitaban áreas de fácil y mediano acceso se extinguieron localmente debido a la fragmentación del hábitat y la sobreexplotación por la caza (Mena et al., 2000). Además, algunos primates y aves huyen cuando los asentamientos humanos se vuelven demasiado grandes o sedentarios (Rival, 2001). Esto coincide con el estudio de Mena et al. (2000) realizado en Keweriono. Los autores informaron que la caza no es sostenible para ningún primate; por ejemplo, el género Ateles tiende a abandonar áreas donde los cazadores se aventuran, mientras que Lagothrix permanece en las mismas áreas, siendo presa fácil de capturar (Papworth, Milner - Gulland y Slocombe, 2013). Mena y Stallings (2000) también informaron que en Keweriono la caza es indiscriminada, y están cazando más individuos por especie y por unidad de tiempo que en otras comunidades. En este estudio, esta comunidad registró el menor número de animales y especies cazados. El uso de armas como escopetas y machetes fue mayor en comunidades de fácil y mediano acceso; las armas son fáciles de transportar y manejar, les permiten moverse rápidamente a través del bosque, son ligeras y pueden matar animales arbóreos y terrestres en mayor número. Con armas de fuego, se cazó $807,86 \mathrm{~kg}$. de biomasa animal equivalente al $59 \%$ de las capturas. Sin embargo, el uso de armas tradicionales fue mayor en Bameno (28,9\%); Cazar con cerbatana representa la elección de una relación cercana con las especies arbóreas. Los Waorani pueden soplar más de diez flechas y matar a un mono en media hora (Rival, 1996), pero con una escopeta, en media hora, los cazadores pueden matar a todo un grupo de monos. Las lanzas y las cerbatanas están hechas completamente de productos del bosque, mientras que la obtención de armas de fuego y machetes depende de los recursos monetarios. Por ejemplo, el precio de una munición varía entre 3 y 3.50 USD, el costo de una sola arma de fuego oscila $\$ 150$, y un machete cuesta US \$ 5. Si el cazador dispara y falla, perdería alrededor de US \$ 3 por munición. Estos datos coinciden con los estudios realizados por (Cueva, et al., 2004 y Mena-Valenzuela et al., 1997; Mena et al., 2000) sobre el aumento en el uso de armas de fuego para cazar. En consecuencia, es probable que los cazadores de comunidades aisladas cacen con armas tradicionales. Los cazadores les apetecen los pecaríes, debido a su sabor, carne blanda y grasosa (Rival, 2001), y por el tamaño o la facilidad de obtenerlo (Mena et al., 2000). En Bameno, la comunidad de acceso difícil, el $71 \%$ de cazadores les apetece el pecarí de labio blanco; en esta comunidad, los pecaríes de labio blanco se cazaron con mayor frecuencia que en el resto de comunidades. Con respecto a las 
comunidades de fácil acceso, en Ñoneno, el 57\% les apetece el pecarí de labio blanco; sin embargo, en esta comunidad se capturaron más guatusas y, en Tiwino, el 70\% les apetece el pecarí de collar, éste fue el más cazado. En Keweriono, la comunidad de acceso medio, el $50 \%$ les apetece el mono lanudo; sin embargo, en esta comunidad, cazaron más aves chachalacas. Factores como la abundancia de ciertos animales y el tiempo de fructificación de ciertas especies vegetales, debe considerarse. Por ejemplo, en abril hay más densidad de fruta y las pavas son atraídas; mayo es el período de engorde del mono lanudo (Rival, 1996; Papworth, Milner-Gulland y Slocombe, 2013). Octubre y noviembre coinciden con la maduración de la fruta de la palmera Moretal (Mauritia flexuosa) y la búsqueda de pecaríes podría ser más fácil (Franzen, 2006; Rival, 1996). Además, la distribución de especies está relacionada con la preferencia y el desplazamiento del hábitat, por ejemplo, los pecaríes de labio blanco caminan en grupos de más de 50 individuos y recorren distancias de hasta 10 km. por día (Tirira, 2017); por esta razón, necesitan bosques bien conservados como en Bameno. Mientras que pecaríes de collar caminan por senderos pequeños y forman grupos de 3 a 9 individuos y el macho adulto puede ser solitario (Tirira, 2007); Tiwino y Noneno, al ser comunidades de fácil acceso, han alterado los bosques donde la probabilidad de encontrar pecaríes de collar es mayor. Mientras, Keweriono no registra la presencia de pecaríes porque las áreas de saladeros están muy lejos de la comunidad.

Con respecto al segundo objetivo sobre las frecuencias de consumo de proteína animal; el consumo de carne de monte fue mayor en Bameno, mientras que, en comunidades de acceso medio y fácil, el consumo de carne de monte fue menor. En Bameno se come 3 días/semana. En Noneno, fue 2 días/semana; en el pasado, esta comunidad dependía de la venta de madera y tráfico de animales (Aguirre, 2007); actualmente, consumen roedores como guantas, guatusas y guatines según (Bodmer, Fang, Moya y Gill, 1994) éstos pueden habitar bosques degradados y soportar presiones de cacería. En Keweriono, el consumo de carne de monte fue de 1día/semana; esta comunidad tiene una historia de cacería no sostenible en el tiempo (Mena-Valenzuela et al., 1997; Mena et al., 2000). En Tiwino, el consumo de carne de monte fue de 1 día/semana; esta comunidad está situada al final de la Vía Auca; en el pasado, es posible que Tiwino haya comercializado carne del monte.

El consumo de pescado silvestre en Bameno y Keweriono fue 3 días/semana. En Bameno, a la gente le gusta alternar carne de monte con pescado silvestre; en Bameno hay numerosos ríos y arroyos que rodean la comunidad. Mientras que en Keweriono, la pesca es la actividad principal debido a la extinción local de especies silvestres (Mena-Valenzuela et al., 1997; Mena et al., 2000); además, en Keweriono, la mayoría de los cazadores trabajan en turismo, y otros estudian porque tiene una unidad educativa donde las personas pueden estudiar en la escuela primaria y secundaria. En Tiwino y Ñoneno se consume pescado silvestre 2 días/semana; en esta comunidad, se observó que las familias iban a pescar de noche; además, en Tiwino, el pescado es parte de su dieta. De todas las comunidades estudiadas, Tiwino fue el único que tenía piscícolas. En Keweriono y Tiwino, los productos enlatados se consumen 
1 día/semana. Se debe tener en cuenta los ingresos económicos y la accesibilidad permiten a los habitantes comprar productos enlatados. En Tiwino, el 95\% de los jefes de hogar tiene empleo remunerado, y en Keweriono el 90\%. En Bameno y Ñoneno, el consumo de productos enlatados no se registró. Además, en estas comunidades, el porcentaje de empleo remunerado fue menor.

En comunidades de fácil acceso, la ingesta de carne comprada es mayor que la de las comunidades de acceso medio y difícil. En Tiwino, la carne comprada se consume 2 días/semana y en Ñoneno 1 día/semana; estas comunidades no están lejos de la ciudad "El Coca" y comprar pollo o pescado fresco es una alternativa para complementar la proteína animal. Además, el precio por libra de carne blanca es más bajo que comprar una munición para armas de fuego. Se debe considerar que las horas que trabajan en empleos remunerados les limita para ir de cacería. Ante esta situación, ellos prefiriesen comprar carne o enlatados. En Bameno y Keweriono, no se registró el consumo semanal de carne comprada; podría deberse a la accesibilidad difícil y mediana; en esas comunidades su alimentación se complementa con almidones obtenidos de cultivos de huerta, principalmente yuca (Manihot esculenta), plátano (Musa paradisiaca) y batata (Ipomoea batatas).

\section{Conclusión}

- La cantidad de kilogramos (biomasa animal), el tipo de especie, el uso de armas y el animal apetecible varían en relación con el nivel de acceso de una comunidad a El Coca; la comunidad de difícil acceso consumió mayor cantidad de biomasa animal silvestre, tuvo diferentes especies de primates y aves, mayor porcentaje de uso de cerbatana y consumieron más ungulados.

- Las frecuencias de consumo de carne de monte y carne comprada varían en relación con la accesibilidad; en Bameno, comunidad de difícil acceso, el consumo de carne de monte fue mayor en relación con las otras comunidades; mientras que el consumo de carne comprada fue mayor en Tiwino y Ñoneno, comunidades de fácil acceso, la carne de pollo o pescado fresco se adquiere. Las frecuencias de consumo de pescado silvestre y de enlatados no varían con la accesibilidad. La frecuencia de consumo de enlatados depende de los ingresos monetarios que las familias perciban semanalmente y la frecuencia de consumo de pescado silvestre dependerá de las condiciones hidrográficas que tenga cada comunidad.

- En el pasado los patrones de movilidad de los pobladores amazónicos se daba por la falta de proteína animal, actualmente, se observa que estos patrones de movilidad han cambiado a lo largo del tiempo, las comunidades se han vuelto sedentarias, y las 
familias son motivadas a vivir cerca de lugares donde el acceso a recursos monetarios sea seguro y otras familias se han movilizado para estar cerca de mercados para abastecerse de productos que les permitan suplir su dieta con proteínas alternativas y otros suplementos.

\section{Referencias}

Aguirre, M. (2007). ¡ A quién le importan esas vidas!: un reportaje sobre la tala ilegal en el Parque Nacional Yasuní.

Bass M.S., Finer M., Jenkins C.N., Kreft H., Cisneros-Heredia D.F., McCracken S.F., et al. (2010). Global Conservation Significance of Ecuador's Yasuní National Park. PLoS ONE 5(1): e8767. https://doi.org/10.1371/journal.pone.0008767

Bodmer, R. E., Fang, T. G., Moya, L., \& Gill, R. (1994). Managing wildlife to conserve Amazonian forests: population biology and economic considerations of game hunting. Biological conservation, 67, 29-35.

Borgerson, C. (2015). The effects of illegal hunting and habitat on two sympatric endangered primates. International Journal of Primatology, 36(1), 74-93.

Bryja, G. (2009). Análisis de las presiones antropogénicas sobre biodiversidad en la Reserva de Biósfera Yasuní. In: Quito.

Cabodevilla, M. Á. (1995). Los" huaorani" en la historia de los pueblos de oriente: Provincia PP Capuchinos de Navarra, Cantabria y Aragón.

Cueva, R., Ortiz, A., \& Jorgenson, J. (2004). Cacería de fauna silvestre en el área de amortiguamiento del Parque Nacional Yasuní, Amazonía Ecuatoriana. Wildlife Conservation Society-WCS. Memorias del VI Congreso Internacional sobre Manejo de Fauna Silvestre en la Amazonía y Latinoamérica. Iquitos, Perú: Universidad Nacional de la Amazonia Peruana (UNAP), Durrell Institute of Conservación and Ecology (DIC.

Da-Gloria, P., \& Bueno, L. (2018). 7 Biocultural Adaptation and Resilience in the HunterGatherers of Lagoa Santa, Central-Eastern Brazil. Hunter-Gatherer Adaptation and Resilience: A Bioarchaeological Perspective, 81(15), 141.

Da Silva Neto, B. C., do Nascimento, A. L. B., Schiel, N., Alves, R. R. N., Souto, A., \& Albuquerque, U. P. (2017). Assessment of the hunting of mammals using local ecological knowledge: an example from the Brazilian semiarid region. Environment, development and sustainability, 19(5), 1795-1813

Franzen, M. (2006). Evaluating the sustainability of hunting: a comparison of harvest profiles across three Huaorani communities. Environmental Conservation, 33, 36-45.

Franzen, M., \& Eaves, J. (2007). Effect of market access on sharing practices within two Huaorani communities. Ecological Economics, 63(4), 776-785. 
International Union for Conservation of Nature. (2018). IUCN Red List of Threatened Species. Retrieved from https://www.iucn.org/resources/conservation-tools/iucnred-list-threatened-species

Jorgenson, J. P., \& Rodríquez, M. C. (2001). Conservación y desarrollo sostenible del Parque Nacional Yasuní y su área de influencia: memorias del seminario-taller 2001: Editorial Simbioe.

Lara, R., Pichilingue, E., Narváez, R., Moreno, P., Sánchez, G., \& Hernández, P. (2002). Plan de Manejo de Territorio Huaorani. Quito: Proyecto CARE/SUBIR, EcoCiencia y ONHAE.

Lu, F. E. (2010). The Conservation Catch-22: Indigenous Peoples and Cultural Change 7987. In Human Ecology: Contemporary Research and Practice. Springer 2010.

Lu, F. E. (2001). The common property regime of the Huaorani Indians of Ecuador: Implications and challenges to conservation. Human Ecology, 29(4), 425-447.

McMullan, M., \& Navarrete, L. (2013). Fieldbook of the Birds of Ecuador: including the Galápagos Islands.

Mena-Valenzuela, P., Regalado, J., \& Cueva, R. (1997). Oferta de animales en el bosque y cacería en la comunidad huaorani de Quehueire'ono, zona de amortiguamiento del Parque Nacional Yasuní, Napo, Ecuador. Estudios biológicos para la conservación: diversidad, ecología y etnobiología. EcoCiencia, Quito, 395-426.

Mena, P., \& Cueva, R. (2001). Cacería de Subsistencia en tres Comunidades del Área de Amortiguamiento del Parque Nacional Yasuní. Conservación y desarrollo sostenible del Parque Nacional Yasuní y su área de influencia, eds. JP Jorgenson y M. Coello Rodríguez. Ministerio del Ambiente/UNESCO/Wildlife Conservation Society. Editorial Simbioe. Quito, Ecuador, 101-127.

Mena, V. P., Stallings, J. R., Regalado, J. B., \& Cueva, R. L. (2000). The sustainability of current hunting practices by the Huaorani. Hunting for sustainability in tropical forests, $57-78$.

Ortiz-von Halle, F. (2011). La selva silenciosa: diez motivos por los que la pérdida de la fauna por cacería insostenible es un serio problema ambiental y social. In K. A. \& M. Mora (Eds.), Ritos y Amenazas del Yasuní (pp. 117). Quito.

Papworth, S., Milner-Gulland, E., \& Slocombe, K. (2013). The natural place to begin: the ethnoprimatology of the Waorani. American Journal of Primatology, 75(11), $1117-$ 1128.

Quirós-Fernández, F., Marcos, J., Acevedo, P., \& Gortázar, C. (2017). Hunters serving the ecosystem: the contribution of recreational hunting to wild boar population control. European journal of wildlife research, 63(3), 57.

Redford, K. H., \& Robinson, J. G. (1987). The game of choice: patterns of Indian and colonist hunting in the Neotropics. American anthropologist, 89, 650-667.

Rival, L. (1992). Huaorani y petróleo. G. Tassi, Náufragos del mar verde. Quito: Abya Yala. 
Rival, L. (2001). Cerbatanas y lanzas. La significación social de las elecciones tecnológicas de los Huaorani. Naturaleza y Sociedad. Perspectivas antropológicas. México: Editorial Siglo XX, 169-191.

Rival, L. M. (1996). Hijos del sol, padres del jaguar: los huaorani de ayer y hoy.

Robinson, J. and K. Redford (1991). Subsistence and commercial uses of wildlife in Latin America. Neotropical Wildlife Use and Conservation., The University of Chicago Press. Chicago.

Stafford, C. A., Preziosi, R. F., \& Sellers, W. I. (2017). A pan-neotropical analysis of hunting preferences. Biodiversity and Conservation, 26(8), 1877-1897.

Stefanik, P. A., \& Trulson, M. F. (1962). Determining the frequency intakes of foods in large group studies. The American journal of clinical nutrition, 11(5), 335-343.

Suárez, E., Zapata-Ríos, G., Utreras, V., Strindberg, S., \& Vargas, J. (2013). Controlling access to oil roads protects forest cover, but not wildlife communities: a case study from the rainforest of Yasuní Biosphere Reserve (Ecuador). Animal Conservation, 16(3), 265-274.

Tirira, D. (2007). Mamíferos del Ecuador: Guía de campo (Vol. 6): Ediciones Murciélago Blanco.

Tirira, D. (2017). Guía de Campo de los Mamíferos del Ecuador. Ediciones Murciélago Blanco. Publicación especial sobre los mamíferos del Ecuador (2nd ed.). QuitoEcuador: Murcielago Blanco.

Urgilés, C. (2006). Estimación de la Sustentabilidad de la cacería de subsistencia en Cuatro Shuar de la asociación Mankusas, estribaciones orientales de la cordillera del Kutukú, Morona Santiago. Universidad Central del Ecuador, Quito.

Valencia, J. H., Toral, E., Morales, M., Betancourt, R., \& Barahona, A. (2008). Guía de campo de reptiles del Ecuador. Fundación Herpetológica Gustavo Orcés, Simbioe, Quito, Ecuador.

Vickers, W. T. (1989). Los sionas y secoyas: su adaptación al ambiente amazónico. 9.

Villaverde, X. (2005). Parque Nacional y Reserva de Biosfera Yasuní: historia, problemas y perspectivas.

Zapata-Ríos, G., Suárez, E., Utreras, B. V., \& Vargas, J. (2006). Evaluation of anthropogenic threats in Yasuní National Park and its implications for wild mammal conservation. Lyonia, 10, 47-57.

Zapata Ríos, G., Toasa, G., Neill, D. A., \& Jørgenson, J. P. (2004). Los pueblos indígenas y el manejo de fauna silvestre: El caso de los Awá y Shuar del Ecuador. Memorias: VI Congreso Internacional sobre Manejo de Fauna Silvestre en la Amazonia y Latinoamérica. 5-10 septiembre 2004, Iquitos-Perú.

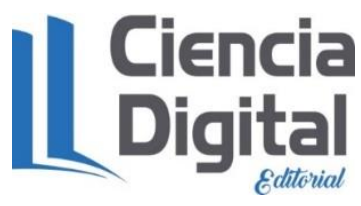




\section{PARA CITAR EL ARTÍCULO INDEXADO.}

Bayancela Delgado, S. (2019). Patrones de cacería y consumo de proteína animal en cuatro comunidades Waorani, Reserva de Biósfera Yasuní. Ciencia Digital, 3(3.2.1), 43-60. https://doi.org/10.33262/cienciadigital.v3i3.2.1.781

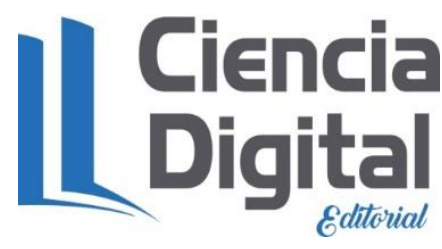

El artículo que se publica es de exclusiva responsabilidad de los autores y no necesariamente reflejan el pensamiento de la Revista Ciencia Digital.

El artículo queda en propiedad de la revista y, por tanto, su publicación parcial y/o total en otro medio tiene que ser autorizado por el director de la Revista Ciencia Digital.
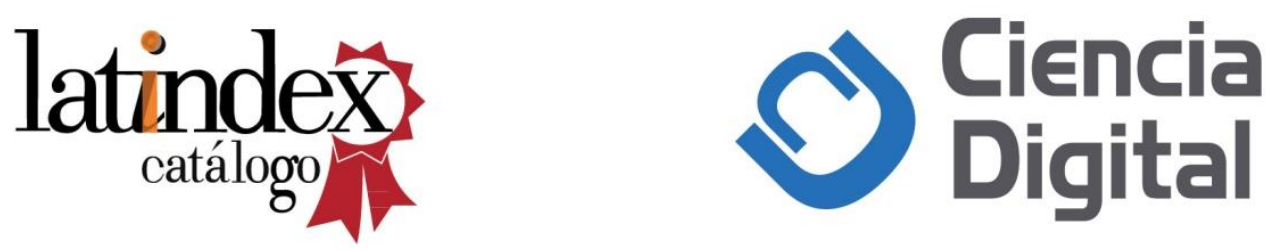